\section{Sea-ice decline due to more than warming alone}

SIR - The dramatic loss of sea-ice cover over the Arctic this summer was widely reported, for example in your News story 'Arctic melt opens Northwest passage' (Nature 449, 267; 2007), and frequently attributed to global warming. Although the gradual decline in sea-ice extent during the past four decades is in line with that expected from global warming, it is very unlikely that the loss of sea-ice cover this year is explicable solely in terms of temperature change.

Changing wind patterns are an important influence on the distribution of sea ice. Throughout summer 2007, exceptional pressure and wind patterns persisted over the Arctic Ocean. The observed migration of ice cover, from the Siberian and Beaufort seas northwards and eastwards into the Arctic Basin, was in line with the expected response to the anomalous winds. These Arctic wind anomalies were part of a global-scale pattern of highly unusual circulation this summer, the causes of which are as yet unclear.

The growing La Niña in the East Pacific undoubtedly had a major influence globally, and there is some evidence from past events that La Niña predisposes the circulation towards the type of exceptional patterns seen this summer.

\section{Julia Slingo, Rowan Sutton}

National Centre for Atmospheric Science, Walker Institute for Climate System Research, University of Reading, Earley Gate, Reading RG6 6BB, UK

\section{Ethics reviews can be centralized without delays}

SIR - The problems inherent in continuing the current system of local ethics reviews for multicentre trials are appropriately highlighted in your News Feature 'Trial and error' (Nature 448, 530-532; 2007). However, you state that the Central Institutional Review Board of the US National Cancer Institute (NCI) adds bureaucracy by its efforts to centralize review. We disagree.

A unique feature of our model is that both central and local boards can be involved; the extent of the local review remains the prerogative of the local board, and their task is facilitated by access to the central board's review. This model has had widespread acceptance: $66 \%$ of NCI-designated cancer centres have now joined. The institute set up a central board for adult and paediatric cancer treatment trials because of the redundancy that occurs when each site's local institutional review board performs a separate ethics review. For example, 50 to 200 sites typically participate in NCI-sponsored phase III trials.

We do not agree that this centralization delays the time it takes to activate a study. The institute's Cancer Therapy Evaluation Program reveals that, for more than two-thirds of the protocols reviewed by the central board, activation is not delayed, as numerous other activities continue in parallel, such as creation of case report forms, finalization of contracts with pharmaceutical partners and drug shipment plans.

We believe that centralized ethics review, with local context supplied by the local board, is the preferred way to conduct review of multisite protocols. The NCI is currently supporting a formal cost analysis by an independent third party. In view of the high local costs cited in your News story, such data should compel administrators of local boards to reconsider their position on centralized review.

Jeffrey Abrams, Jacquelyn Goldberg Central Institutional Review Board Initiative, National Cancer Institute, 6130 Executive Boulevard, Bethesda, Maryland 20892, USA

\section{Missing the mark on biomedical research}

SIR - Brian Martinson in his Commentary 'Universities and the money fix' (Nature 449, 141-142; 2007) suggests that short-sighted greed for federal grants motivates academic leadership to sacrifice the future interests of biomedical research. On the contrary, such grants do not cover the full costs of the research projects they support.

The annual shortfall of billions of dollars (see C. A. Goldman and T. Williams Paying for University Research Facilities and Administration Rand, Santa Monica; 2000) must be offset from the limited sources of discretionary funds available to universities: tuition fees, state funding, philanthropy, technology transfer or revenues derived from the clinical practice of the faculty.

Of these, philanthropy is serendipitous and generally attracts investment in new initiatives rather than in sustaining existing programmes. The pressures on tuition costs and clinical-practice margins have been widely publicized. Technology transfer only rarely brings significant, sustainable revenues.

If academic institutions merely sought to maximize short-term revenues, they would not make the substantial long-term commitments of capital to physical plant, state-of-the-art technologies and skilled personnel that they do.

We believe that the expansion of research capacity is being driven instead by the explosive pace of advances in the biomedical sciences and in our understanding of the processes of health and disease, as well as by mounting dependence on multidisciplinary research teams and increasingly sophisticated new technologies.
These investments are inherently risky in their anticipation of future returns from federal and other sponsored research. Accordingly, the decision-making behind them is deliberate and calculated: university governing boards, bound by their fiduciary obligations, are inherently conservative.

The distress caused by National Institutes of Health budgets that have steadily declined in purchasing power since 2003 is being widely and acutely felt. But increases in these budgets are unlikely; simply sustaining the enterprise to keep pace with inflation which Martinson terms "prudent" has been difficult.

The biomedical research enterprise does need to adapt to fiscal realities, but restructuring the historic 'business model' for academic research, including the composition of its workforce, will be very difficult. At minimum, such restructuring must be guided by sensitivity to the institutions' missions, recognition of the intense sociopolitical expectations of them and accurate perception of what is necessary for their academic and economic survival. David Korn, Stephen J. Heinig Association of American Medical Colleges, 2450 N Street, Washington DC 200037, USA

\section{Words of wisdom worth reading aloud}

SIR - I congratulate Erez Lieberman and colleagues on their entertaining, enthralling and, above all, well-written Letter 'Quantifying the evolutionary dynamics of language' (Nature 449, 713-716; 2007).

Nature articles outside my sphere of interest are of interest to me as far as the abstract; after that I am rapidly lost in a forest of jargon, technicalities and poorly articulated background information.

I had little prior interest in linguistic evolution, yet Lieberman and colleagues captured my attention to the end of their Letter. It was so well written that I was compelled to read out the cleverest snippets to my office-mates.

This is a scientific paper that has truly exemplified the aim of Nature to provide a forum for work with interdisciplinary appeal. I congratulate and thank both the authors and the selecting editors.

Vanessa S. Solomon

Peter MacCallum Cancer Centre, St Andrew's Place, East Melbourne, Victoria, Australia

\footnotetext{
Contributions to Correspondence may be submitted to correspondence@nature.com. They should be no longer than $\mathbf{3 0 0}$ words, and ideally shorter. They should be signed by no more than three authors; preferably by one. Published contributions are edited.
} 